\title{
ENSINO DA HABILITAÇÃO EM ENFERMAGEM MEDICO-CIRÚRGICA DA ESCOLA DE ENFERMAGEM DA UNIVERSIDADE DE SÃO PAULO - Análise do programa de Enfermagem Médico-Cirúrgica II desenvolvido em 1976.
}

Yoriko Kamiyama*
Célia Pires de Araújo*
Moema Guedes Barbato*
Maria Sumie Koizumi
Coracy Brochini da Graça Martins*
Miako Miura*
Edna Ikumi Umebayashi

KAMIYAMA, Y.; ARAÛJO, C. P.; BARBATO, M. G.; KOIZUMI, M. S.; MARTINS, C. B. G.; MIURA, M.; UMEBAYASHI, E. I. - Ensino da Habilitação em Enfermagem Médico-Cirúrgica - Análise do programa de Enfermagem Médico-Cirúrgica II desenvolvido em 1976. Rev. Esc. Enf. USP, 11(1):51-69, 1977.

Os autores analisam o programa de Enfermagem MédicoCirúrgica II da EEUSP desenvolvido em 1976. Com base na avaliação feita por docentes, alunos e enfermeiros dos campos de estágio, concluem que o programa parece estar atendendo à sua finalidade principal que é a de contribuir na formação de enfermeiros com preparo especializado para eficiente atuação nas unidades de terapia intensiva, de recuperação $e$ de pronto socorro.

\section{INTRODUÇÃO}

Em trabalho anterior abordaram-se os aspectos gerais da disciplina Enfermagem Médico-Cirúrgica II da EEUSP (EMC II), intro-

* Docente da Disciplina Enfermagem Médico-Cirúrgica II, do Departamento de Enfermagem Médico-Cirúrgica da Escola de Enfermagem da USP. 
duzida em 1973 como integrante da habilitação em Enfermagem MédicoCirúrgica (Parecer n. ${ }^{\circ}$ 163/72 do CCR. de Currículos do C.F.E.), com descrição especial do programa desenvolvido em 1976. (KOIZUMI et al, 1976).

O referido programa, por conter expressivas modificações em relação aos anteriores e por representar o fruto de quatro anos de experiência, foi avaliado formalmente não só pelo corpo docente e discente mas também pelos enfermeiros dos campos de estágio. A avaliação foi feita com base nos objetivos do programa, ou seja, tendo terminado a disciplina e atuado, então em unidades de terapia intensiva, unidades de recuperação e pronto socorro, o aluno deverá ser capaz de:

- discorrer sobre a etiologia, a fisiopatologia, o tratamento e as complicações das coronariopatias, da insuficiência renal aguda, dos estados de choque, da insuficiência respiratória aguda, dos politraumatizados e das afeç̧ões cirúrgicas do coração, dos vasos da base e neurocirúrgicas;

- fazer, interpretar e registrar exames complementares específicos;

- executar o processo de enfermagem em pacientes agudos graves;

- manejar e controlar aparelhos usados nas referidas unidades: monitores, desfibriladores, marca-passos, respiradores, aspiradores e hemodializadores.

No presente trabalho, procura-se relatar como foi feita essa avaliação e quais os resultados obtidos.

\section{AVALIAÇÃO FEITA PELOS DOCENTES}

Durante a execução do programa, realizaram-se reuniões semanais para revisão do planejamento e avaliação. Nelas, eram analisadas as atividades desenvolvidas na semana anterior, discutidos os problems relativos ao ensino teórico-prático e ao estágio. Com base nessa 
avaliação eram mantidas ou replanejadas as atividades para a semana seguinte.

O programa do ano de 1976 incluiu algumas modificações: ensinos clínicos com nova abordagem; inclusão de trabalhos de iniciação à pesquisa; escolha dos serviços especializados para visita, bem como organização dos grupos, determinação de datas e horários, feita pelos próprios estudantes; inclusão da Unidade de Terapia Intensiva de Tetânicos como um dos campos de estágio relativos à Unidade Respiratória; modificações no sistema e nos instrumentos de avaliação do estágio e dos trabalhos apresentados pelos alunos. Para verificar a validade dessas modificações e com o intuito de obter dados para melhoria do ensino, julgou-se oportuno avaliá-lo formalmente.

Elaborou-se um questionário (Anexo 1) com base nos objetivos do programa, que foi preenchido pelas seis docentes que integraram a ensino teórico-prático e o estágio. Os resultados dessa avaliação podem ser vistos na Tabela 1.

\section{TABELA 1}

Distribuição dos docentes segundo o grau e as dimensões avaliadas da disciplina EMC II, 1976.

\begin{tabular}{l|r|r|c|c}
\hline $\begin{array}{l}\text { Dimensoes } \\
\text { avaliadas }\end{array}$ & $\begin{array}{c}\text { Satisfa- } \\
\text { tório }\end{array}$ & Regular & $\begin{array}{c}\text { Não satis- } \\
\text { tório }\end{array}$ & Total \\
\hline $\begin{array}{l}\text { Conteúdo programático } \\
\text { Estratégias de ensino }\end{array}$ & 6 & - & - & 6 \\
Sistema de avaliação da teoria (prova) & 1 & 5 & - & 6 \\
$\quad \begin{array}{l}\text { Rodízio programado para o } \\
\text { aluno }\end{array}$ & 6 & & & 6 \\
$\quad \begin{array}{l}\text { Período de estágio em cada } \\
\text { UTI }\end{array}$ & 5 & 1 & - & 6 \\
$\quad$ Ensino de campo & 4 & 2 & - & 6 \\
Sistema de avaliação & 5 & 1 & - & 6 \\
\hline
\end{tabular}


De modo geral, podemos considerar positivo o ensino da disciplina como demonstra a Tabela 1 . A maioria das dimensões propostas para mensuração foi avaliada satisfatoriamente.

\section{Conteúdo programático}

O conteúdo programático desenvolvido parece ter proporcionado ao aluno conhecimentos fundamentais sobre a fisiopatologia e a assistência de enfermagem a pacientes em situações críticas que exigem cuidados intensivos e habilidade no seu atendimento. Isso pode ser constatado tanto pelo rendimento teórico demonstrado pelos alunos (nota média 8,6), quanto pelo seu desempenho no campo clínico (nota média 9,0).

\section{Estratégias de ensino}

Todas as estratégias de ensino, isto é, preleção, discussão em grupo, estudo dirigido, demonstração, seminário, visita a serviços especializados e ensinos clínicos, foram consideradas válidas. Os "ensinos clínicos renovados" (ARAÚJO et al, 1977), elaborados sob orientação formal de um professor orientador e avaliados por uma junta de dois docentes, foram considerados como uma experiência altamente positiva. Foram apontadas como principais vantagens desse método de ensino: estimular o desenvolvimento integral do aluno; proporcionar uma visão global das unidades de terapia intensiva, e oferecer oportunidade de realização de pesquisas.

\section{Estágio}

O ensino de campo foi contínuo e individualizado, segundo as necessidades de cada estudante. Assim, no estágio, cada docente orientava de 2 a 4 estudantes.

O estágio foi considerado satisfatório, exceto em algumas unidades onde $o$ pequeno número de pacientes parece ter prejudicado, em parte, a aprendizagem do aluno.

Houve constante preocupação em dirigir e estimular os alunos a se desenvolverem na assistência global ao paciente agudo grave e levá-los à conscientização dos problemas de ordem geral vigentes 
nas unidades de terapia intensiva. As diretrizes básicas do ensino de campo foram delineadas em reuniōes da disciplina. Os instrumentos para o levantamento de dados do paciente e planejamento de cuidados utilizados pelos alunos foram comuns em todas as unidades. (KOIZUMI et al. 1976 e KOIZUMI, 1976).

Ainda quanto ao estágio, uma das atividades docentes mais difíceis foi a avaliação do desempenho do aluno, por envolver mensuração de atitudes, além de conhecimentos e habilidades.

MILLER (1967), analisando a problemática da avaliação do desernpenho dos estudantes de Medicina no campo clínico, comenta que a técnica de observação, mesmo com algumas desvantagens que implicam em subjetividade, ainda é uma das melhores. A subjetividade pode ser sensivelmente diminuída quando a filosofia da disciplina, os seus objetivos e critérios de avaliação são pré-determinados e, além disso, quando são empregados formulários específicos para o registro das observações.

No caso, a avaliação do estudante foi contínua durante toda a prática no hospital, mediante a técnica da observação participante e não participante com uso do instrumento específico (Anexo 2). Esse instrumento de avaliação foi preenchido pelo aluno e pelo professor, seguindo os mesmos critérios, ao término da prática em cada unidade (Anexo 3). A avaliação final (Anexo 4) constou da média aritmética dos conceitos revertidos em notas, que foram obtidas pelo aluno em cada unidade.

$\mathrm{Na}$ elaboração dos instrumentos de avaliação, procurouse utilizar, tanto quanto possível, os princípios gerais da avaliação da experiência clínica; no entanto, alguns itens, sobretudo os referentes a atitudes, permaneceram subjetivos, como observaram dois docentes.

\section{Prova de aproveitamento}

O grande volume de matéria do bloco teórico (120 horas) fez com que se organizasse a prova em forma de teste com 100 questões para que se incluísse pelo menos uma questão para cada hora-aula. Foram concedidas 3 horas para o seu preenchimento. Essa experiência 
foi considerada regular, pois, embora fosse bom o conteúdo da prova, o número excessivo de questões tornou-a cansativa para os alunos.

Ainda na tentativa de elucidar melhor o nível de rendimento discente, pode-se mencionar a nota média da classe - na teoria $(8,6)$ e na prática $(9,0)$ - já citada anteriormente.

A porcentagem de freqüência às aulas e ao estágio foi também bastante elevada, $97,0 \%$ e $94,0 \%$, respectivamente, o que parece reforçar a idéia de que o curso suscitou e manteve o interesse nos alunos, motivando-os a um bom desempenho nas suas responsabilidades.

\section{AVALIAÇÃO FEITA PELOS ENFERMEIROS DOS CAMPOS DE ESTÁGIO}

Para completar a análise da disciplina EMC II, solicitou-se a avaliação dos alunos aos enfermeiros (10, no total) que os acompanharam na fase final do estágio. Essa avaliação foi feita por meio do formulário (Anexo 5) e os seus resultados são apresentados nas Tabelas 2,3 e 4 .

\section{TABELA 2}

Distribuição dos enfermeiros de campo de estágio, segundo a avaliação feita por eles relativa ao conhecimento dos estudantes, 1976.

\begin{tabular}{|c|c|}
\hline AVALIAÇÃO & N. ${ }^{\circ}$ DE ENFERMEIROS \\
\hline $\begin{array}{l}\text { - conhecem a fisiopatologia e a correlaciona } \\
\text { com a prática } \\
\text { - conhecem a fisiopatologia e necessitam de } \\
\text { pouca complementação teórica para rela- } \\
\text { cioná-la à prática } \\
\text { - têm conhecimentos básicos sobre a fisio- } \\
\text { patologia e necessitam de complementação } \\
\text { teórica e supervisão direta } \\
\text { - têm pouco conhecimento, ou mesmo ne- } \\
\text { nhum, sobre a fisiopatologia }\end{array}$ & $\begin{array}{r}1 \\
-\end{array}$ \\
\hline TOTAL & 10 \\
\hline
\end{tabular}




\section{TABELA 3}

Distribuição dos enfermeiros de campo de estágio, segundo a avaliação feita por eles relativa à habilidade dos estudantes, 1976.

\begin{tabular}{c|c}
\hline \multicolumn{1}{c|}{ AVALIAÇÃO } & N. DE ENFERMEIROS \\
\hline $\begin{array}{l}\text { demonstram habilidade na identificação } \\
\text { de problemas, na elaboração e execução } \\
\text { do plano de cuidados e pronta atuação nas } \\
\text { intercorrências }\end{array}$ & 5 \\
- demonstram habilidade na identificação \\
$\begin{array}{l}\text { de problemas e dificuldade na atuação } \\
\text { nas intercorrências }\end{array}$ \\
$\begin{array}{l}\text { demonstram pouca habilidade na elabo- } \\
\text { ração e execução do plano de cuidado e } \\
\text { dificuldade na atuação nas intercorrências }\end{array}$ \\
\hline \multicolumn{1}{c}{ TOTAL } \\
\hline
\end{tabular}

TABELA 4

Distribuição dos enfermeiros de campo de estágio, segundo a avaliação feita por eles relativa às atitudes dos estudantes, 1976.

\begin{tabular}{|c|c|c|c|c|}
\hline ATITUDES GRAU & Satisfatório & Regular & Não satisfatório & Tota \\
\hline Interesse & 9 & 1 & - & 10 \\
\hline Iniciativa & 3 & 7 & - & 10 \\
\hline Cooperação & 10 & - & - & 10 \\
\hline Estabilidade emocional & 8 & 2 & - & 10 \\
\hline Pontualidade e assiduidade & 7 & 3 & - & 10 \\
\hline Capacidade de comunicação & 6 & 4 & - & 10 \\
\hline
\end{tabular}


Como se observa na Tabela 2, a maioria dos enfermeiros - 8 deles - foi de opinião que os estudantes demonstraram ter conhecimento de fisiopatologia e correlacionaram-na com a assistência prestada ao paciente agudo grave, necessitando de pouca orientação do docente ou do enfermeiro de campo de estágio.

Com relação às habilidades, todos os enfermeiros observaram que os alunos demonstraram habilidade na identificação dos problemas do paciente e na elaboração e execução do plano de cuidados. Porém, quanto à atuação nas intercorrências, conforme mostra a Tabela 3 , cinco dos entrevistados referiram ter identificado nos estudantes pronta atuação, sendo que os outros cinco discordaram, informando que os alunos demonstraram certa dificuldade de atuação.

Esse resultado não é de todo surpreendente pois as intercorrências são as situações mais difíceis para serem solucionadas, sobretudo para os estudantes, que têm pouca vivência no atendimento a pacientes em estado crítico.

No que se refere a atitudes, todos os itens, exceto "iniciativa", foram avaliados como satisfatórios pela maioria dos entrevistados. A iniciativa pouco desenvolvida parece ter influído na dificuldade demonstrada pelos alunos em atuar prontamente nas situações de emergência, já assinalada anteriormente.

Um dos enfermeiros fez a observação de que o aluno seria mais beneficiado no estágio se houvesse melhor entrosamento entre a escola e a unidade campo de estágio.

\section{AVALIAÇÃo FEITA PELOS ALUNOS}

Apresentar-se-á por fim, a avaliação da disciplina feita por escrito pelo próprio estudante.

A coordenadora do curso, quando de seu início, havia solicitado que cada aluno escrevesse as suas expectativas quanto à disciplina EMC II, antes de lhes ser apresentado o programa.

E interessante notar que, de modo geral, as expectativas indicadas pelos estudantes coincidem com os objetivos do curso, como se pode observar na Tabela 5 . 


\section{TABELA 5}

Distribuição dos 20 estudantes ao iniciarem o curso, segundo as suas expectativas em relação à disciplina EMC II.

\begin{tabular}{l|c}
\hline \multicolumn{1}{c|}{ EXPECTATIVAS* } & $\mathrm{N}^{\circ}$ \\
\hline — assistência de enfermagem a pacientes graves de UTI & 13 \\
e em situação de emergência & \\
- aquisição de conhecimentos específicos para atuar junto \\
$\begin{array}{l}\text { a pacientes graves inclusive sobre as condições físicas } \\
\text { ideais das UTI }\end{array}$ & 10 \\
- especialização em conhecimentos e na prática de Enf. & 8 \\
$\begin{array}{l}\text { Médica e Cirúrgica } \\
\text { manipulação de aparelhos especializados auxiliares na } \\
\text { manutenção da vida do paciente ou no diagnóstico }\end{array}$ & 6 \\
- ampliação de conhecimentos sobre patologia para a \\
assistência em Enfermagem \\
outros**
\end{tabular}

As expectativas citadas pelos alunos parecem demonstrar que eles já estavam conscientes do que lhes seria oferecido pela disciplina e da sua própria opção profissional. Isto dá ao docente certa margem de segurança para levar em conta as opiniões dos estudantes ao iniciar a disciplina bem como para compará-las com a avaliação ao seu término.

Apresenta-se agora a avaliação escrita apresentada pelos mesmos 20 alunos, ao final do programa.

* cada aluno citou mais de uma expectativa.

** pesquisa e objetividade no ensino - 1 compreensão, paciência e motivação -3 bom relacionamento entre professor e aluno -1 . 
O conteúdo foi considerado bom, porém com desproporção relativa à divisão das unidades, tendo sido, segundo eles, destinado um número de horas excessivo à unidade de pronto socorro e insuficiente às unidades respiratórias e de choque.

De modo geral, o conteúdo das aulas foi tido como muito bom havendo, no entanto, sugestões para a melhoria de alguns assuntos. A classe foi de opinião que, na medida do possível, as aulas fossem dadas pelos próprios docentes da Escola. Considerou também que os seus conhecimentos anteriores eram insuficientes para acompanhar, com facilidade, o programa teórico desenvolvido.

\section{METODOS UTILIZADOS -}

Foram qualificados como muito proveitosos os ensinos clínicos e as visitas a serviços especializados.

O critério adotado para a escolha do tema dos ensinos clínicos e a designação de orientadoras formais foram salientados como pontos muito válidos. Todavia, a classe sugeriu maior disponibilidade de alguns orientadores e a formação de uma comissão de avaliadores ao invés da junta composta por dois docentes.

A prova única foi considerada positiva, mas algumas questões foram classificadas como subjetivas e para elas foi sugeridas melhor formulação.

\section{ESTÁGIO -}

O período de estágio em cada unidade de terapia intensiva, variando entre duas e três semanas, foị considerado suficiente.

Os campos de estágio foram classificados como muito bons e o rodízio programado para cada grupo, bastante oportuno.

Foi sugerido que se estenda para mais grupos a oportunidade de estagiar em unidade de queimados e unidade de terapia intensiva para crianças. 
A supervisão, segundo os alunos, foi boa por ser à distância e por incluir discussões pós-estágio diárias ou, pelo menos, regulares. Sugeriram que essas discussões sejam mantidas nos próximos programas, se possível diariamente, ao término do estágio.

Como conclusão, a classe julgou o curso muito bom, por terem sido atingidos os objetivos propostos, permitindo amplo desenvolvimento aos alunos.

Notou-se que a avaliação feita pelos alunos, ao término do curso, baseia-se nos objetivos do programa, com análise do conteúdo, das estratégias de ensino, dos trabalhos discentes, do estágio e do sistema de avaliação da aprendizagem teórica. A análise da disciplina parece ter sido feita criteriosamente, o que se vê pela coincidência dos seus pontos de vista com os dos docentes e enfermeiros de campo e por algumas sugestões apresentadas, bastante válidas.

\section{CONCLUSÃO}

Interrelacionando os resultados da avaliação feita pelos docentes, enfermeiros de campo e alunos e confrontando-os com os recursos didáticos disponíveis, chegou-se à conclusão de que o curso atingiu os objetivos propostos, em nível desejado. Isso indica que parece que se está encaminhando para a comunidade enfermeiros capazes de atuar em unidades de terapia intensiva, de pronto-socorro e de recuperação, pelo menos com razoável preparo técnico-científico.

Como foi mencionado, o programa desenvolvido em 1976 é fruto de quatro anos de experiência, sendo que a cada ano vêm-se fazendo alterações. Mesmo assim, o citado programa não é o ideal, apresentando deficiências, como se comentou neste trabalho. A partir da avaliação aqui relatada, serão introduzidas novas modificações, mas é opinião dos docentes manter, em linhas gerais, o mesmo plano de ensino com a reformulação de alguns pontos considerados negativos, e reforço dos que foram pouco enfatizados. 
KAMIYAMA, Y.; ARAÚJO, C. P.; BARBATO, M. G.; KOIZUMI, M. S.; MARTINS, C. B. G.; MIURA, M.; UMEBAYASHI, E. I. Teaching Medical Surgical Nursing in Specialization Course at the School of Nursing of the University of São Paulo - Evaluation about the program developed in 1976. Rev. Esc. Enf. USP, 11(1): 51-69, 1977.

The authors analyse the program of Medical Surgical Nursing II of the School of Nursing of the University of São Paulo, developed in 1976. Based on the evaluation made by teachers, students and nurses of the clinical practice units, they concluded that the program seems to attempt its main finality contributing in nurse's formation with specialized prepare to an eficient action in recovery room and emergency and intensive care units.

\section{REFERENCIAS BIBLIOGRÁFICAS}

ARAÚJO, C. P. et al. - Ensino da habilitação em EMC da EEUSP: uma abordagem renovada dos ensinos clínicos e sua avaliação. Enf. Novas Dimens., 3(2): 1977 (no prelo).

CARVAlHO, Amália C. - Orientação e ensino de estudantes de Enfermagem no campo clínico. São Paulo, 1972. (Tese de doutoramento - Escola de Enfermagem da USP).

KOIZUMI, M. S. - Avaliação de um roteiro para exame físico aplicado em pacientes neurocirúrgicos. Enf. Novas Dimens., 2(4): 222-231, 1976.

KOIZUMI, M. S. et al. - Exame físico em pacientes agudos graves: uma experiência de ensino. Enf. Novas Dimens., 2(5): 284-289, 1976.

KOIZUMI, M. S. et al. - Ensino da habilitação em Enfermagem Médico-Cirúrgica - aspectos da disciplina Enfermagem Médico-Cirúrgica II. Rev. Esc. Enf. USP, 10(3): 331-339, 1976.

MILLER, C. E. - Ensino e aprendizagem nas Escolas Médicas. São Paulo, Nacional, 1967. 


\section{ANEXO 1}

\section{AVALIAÇĀO DA DISCIPLINA ENFERMAGEM MEDICO-CIRÚRGICA II}

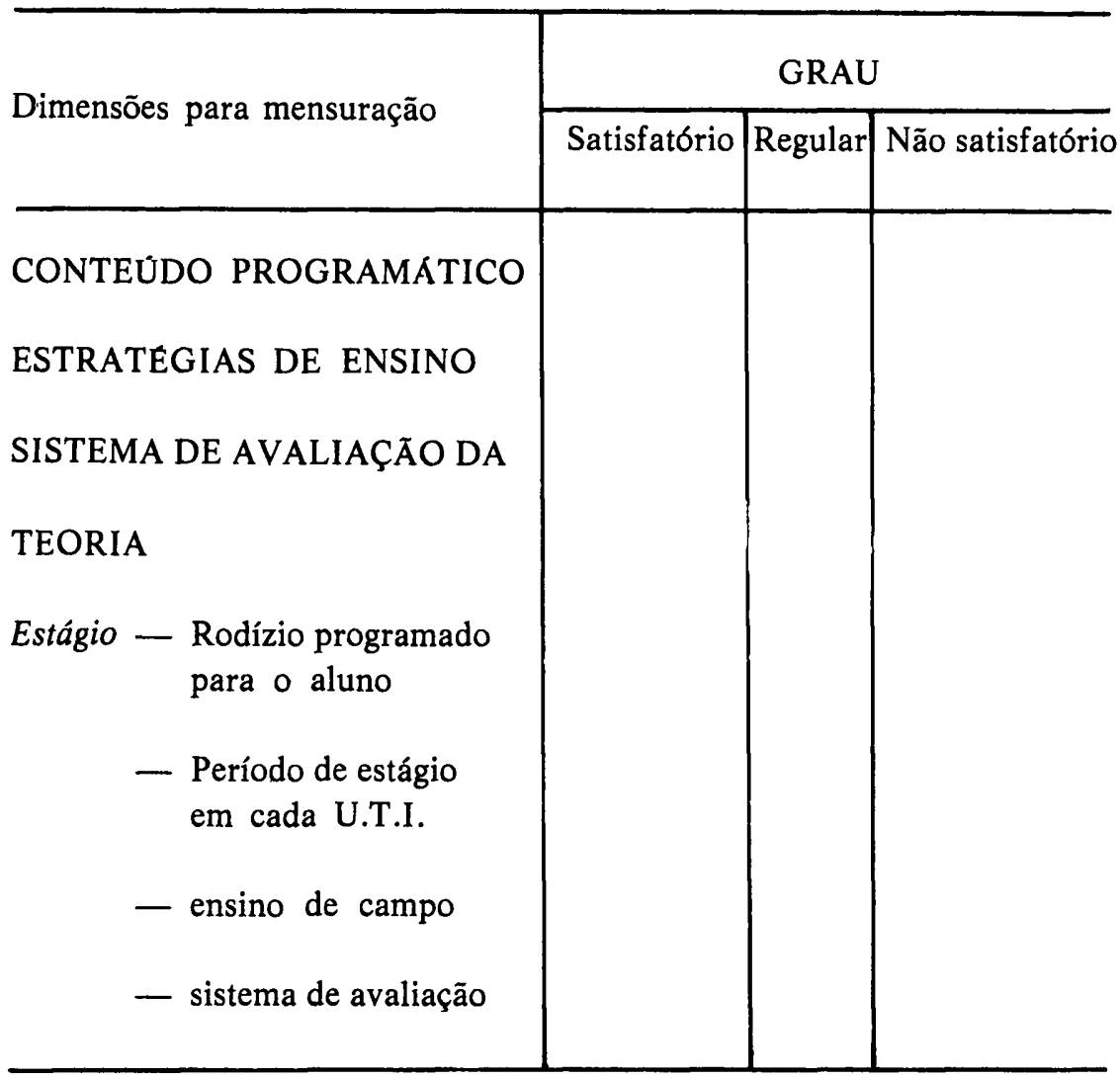

OBSERVAÇŌES E SUGESTOES: 


\author{
ANEXO 2 \\ UNIVERSIDADE DE SÃO PAULO \\ ESCOLA DE ENFERMAGEM \\ AVALIAÇÃO DO ESTÁGIO — EMC II - 1976
}

Unidade

Período

Aluno

Professor

\begin{tabular}{|c|c|c|c|}
\hline & \multicolumn{3}{|c|}{ CONCEITO } \\
\hline & $\begin{array}{l}\text { auto } \\
\text { aval. }\end{array}$ & $\begin{array}{l}\text { aval. } \\
\text { prof. }\end{array}$ & $\begin{array}{c}\text { média } \\
\text { aritmética }\end{array}$ \\
\hline $\begin{array}{l}\text { 1. ÁREA COGNITIVA (peso 4) } \\
\text { Conhecimento da fisiopatologia e sua cor- } \\
\text { relação com a assistência de enfermagem } \\
\text { dada ao paciente. }\end{array}$ & & & \\
\hline $\begin{array}{l}\text { 2. ÁREA PSICOMOTORA (peso 4) } \\
\text { Habilidade: } \\
\text { a) na execução do exame físico } \\
\text { b) no planejamento de cuidados } \\
\text { c) na execução do planejamento } \\
\text { d) em atuar nas intercorrências }\end{array}$ & & & \\
\hline $\begin{array}{l}\text { 3. AREA ÁFETIVA (peso 2) } \\
\text { a) interesse e iniciativa } \\
\text { b) comunicação } \\
\text { c) cooperação } \\
\text { d) estabilidade emocional } \\
\text { e) pontualidade e assiduidade }\end{array}$ & & & \\
\hline
\end{tabular}

MEDIA FINAL DA UNIDADE

CONCEITOS
$\mathrm{A}-10$ a 9
B - 8,9 a 7,5
C $-7,4$ a 5,0
$\mathrm{D}-4,9$ a 0,0 (insuficiente) 


\section{ANEXO 3 \\ UNIVERSIDADE DE SÃO PAULO \\ ESCOLA DE ENFERMAGEM}

CRITERIOS PARA AVALIAÇÃO DO ESTÁGIO — EMC II — 1976

1. AREA COGNITIVA (peso 4)

Conhecimento da fisiopatologia e sua correlação com a assistência de enfermagem dada ao paciente.

A - Conhece a fisiopatologia e faz a correlação com a assistência do enfermeiro dada ao paciente.

B - Conhece a fisiopatologia e necessita de pouca complementação para sua correlação.

C - Conhece o básico da fisiopatologia porém necessita de complementação para fazer a correlação.

D - Pouco ou nenhum conhecimento da fisiopatologia.

2. ÁREA PSICOMOTORA (peso 4)

Habilidade: a) na execução do exame físico

b) planejamento dos cuidados

c) execução do planejamento

d) atuação nas intercorrências

A - Executa os três primeiros itens adequadamente e em tempo hábil. Atua adequadamente na solução das intercorrências.

B - Não demonstra muita habilidade em qualidade ou tempo em um (1) dos itens ( $a, b$ e/ou $c$ ). Atua corretamente na solução das intercorrências mas necessita de orientação.

C - Não demonstra muita habilidade em qualidade $e$ tempo em dois (2) dos três primeiros itens. Pouca atuação durante as intercorrências.

D - Ineficiente nos três itens. Atua desordenadamente nas intercorrências ou não participa das mesmas.

3. AREA AFETIVA (peso 2)

a) Interesse e iniciativa

Demonstra interesse pelos problemas apresentados pelo paciente, procura equacioná-los e tenta resolvê-los. Interessa-se pelo auto 
aprendizagem. Demonstra iniciativa diante das emergências. Apresenta criatividade quando a situação o permite.

b) Comunicação

Comunica-se com eficiência, de maneira segura e clara, com o paciente, familiares, equipe de saúde e de enfermagem.

c) Cooperação

Demonstra espírito de cooperação ajudando, fazendo, esclarecendo, orientando o paciente e familiares e as equipes de saúde e de enfermagem.

d) Estabilidade emocional

Apresenta um controle efetivo das reações (comportamento visível) frente a quaisquer situações de emergência, principalmente aquelas que levam o paciente à invalidez ou morte. Deve demonstrar racionalização frente aos seus problemas e aos apresentados pela equipe de trabalho.

e) Pontualidade e assiduidade

Espera-se do aluno uma pontualidade e assiduidade convenientes, isto é, sem faltas ou atrasos injustificados.

A - Quando o aluno atende aos cinco itens

B - Quando dois itens necessitam ser reforçados

C - Quando três itens necessitam ser reforçados

D - Quando mais de três itens necessitam ser reforçados

CONCEITOS
$A-10$ a 9
B $-8,9$ a 7,5
$\mathrm{C}-7,4$ a 5,0
D $-4,9$ a 0,0

São Paulo, março de 1976. 
ANEXO 4

ESCOLA DE ENFERMAGEM DA USP

BOLETIM DE AVALIAÇĀO

DISCIPLINA: ENFERMAGEM MEDICO-CIRÚRGICA II

Nome:

Período:

MEDIA FINAL:

\begin{tabular}{l|l}
\hline \multicolumn{1}{c|}{ ITENS } & NOTAS \\
\hline 1. ÁREA COGNITIVA & \\
Conhecimento da fisiopatologia e sua correlação com \\
a assistência de enfermagem. & \\
\hline 2. ÁREA PSICOMOTORA & \\
Habilidade: & \\
a) na execução do exame físico & \\
b) no planejamento de cuidados & \\
c) na execução do planejamento & \\
d) em atuar nas intercorrências & \\
\hline 3. ÁREA AFETIVA & \\
a) interesse e iniciativa & \\
b) comunicação & \\
c) cooperação & \\
d) estabilidade emocional & \\
e) pontualidade e assiduidade & \\
\hline
\end{tabular}

OBSERVAÇÕES:

DATA:

DOCENTES

de acordo.

ESTUDANTE 


\section{ANEXO 5}

\section{FORMULARIO}

Este é um trabalho que estamos realizando para avaliar o Programa de Enfermagem Médico-Cirúrgica II - Habilitação da EEUSP - 1976. Para isso queremos contar com a sua colaboração no sentido de responder ao presente formulário. Suas respostas constituirão subsídios para avaliação do programa desenvolvido esse ano e na melhoria dos futuros programas.

\section{IDENTIFICAÇÃO}

Nome: $\ldots \ldots \ldots \ldots \ldots \ldots \ldots \ldots \ldots \ldots \ldots \ldots \ldots \ldots \ldots \ldots \ldots \ldots$ cargo ou função:

U.T.I. Hospital:

Data da entrevista: $\ldots / \ldots / \ldots$ Hora: $\ldots$ Início: $\ldots$ Término: ...

1. Você acha que os estudantes do curso de habilitação em Enfermagem Médico-Cirúrgica que estagiaram nesta unidade:

( ) Conheciam a fisiopatologia e a correlacionavam com a assistência de enfermagem prestada ao paciente.

( ) Conheciam a fisiopatologia e necessitavam de pouca complementação pela docente ou pela enfermeira da UTI, na correlação com a prática.

( ) Conheciam o básico da fisiopatologia porém, para sua correlação com a prática necessitavam de complementação pela docente ou enfermeira da UTI, bem como de supervisão direta e constante.

( ) Tinham pouco conhecimento, ou mesmo nenhum, da fisiopatologia. 
2. Os estudantes demonstraram:

( ) Habilidade na identificação de problemas do paciente, no planejamento dos cuidados, na execução do plano elaborado e pronta atuação nas intercorrências.

( ) Habilidade na identificação de problemas do paciente, no planejamento dos cuidados, na execução do plano e dificuldade na atuação nas intercorrências.

( ) Pouca habilidade na identificação de problemas do paciente, no planejamento dos cuidados, na execução do plano e dificuldade na atuação nas intercorrências.

3. Você observou nos estudantes:

SIM

NÃO

Pouca Regular Muita

\begin{tabular}{|c|c|c|c|c|c|}
\hline ( ) & ( ) & ( & ) & Interesse & () \\
\hline ( ) & () & ( & ) & Iniciativa & ( ) \\
\hline ) & () & ( & ) & Cooperação & ( ) \\
\hline ) & () & ( & ) & Estabilidade emocional & ( ) \\
\hline ) & () & ( & ) & Pontualidade e assiduidade & ( ) \\
\hline ) & () & ( & ) & Capacidade de comunicação & ( \\
\hline
\end{tabular}

OBSERVAÇŌES:

Assinatura do entrevistador 